\title{
SISTEMATIZAÇÃO DA ASSISTÊNCIA DE ENFERMAGEM AO PACIENTE PEDIÁTRICO ONCOLÓGICO EM UM HOSPITAL PÚBLICO NO INTERIOR DA AMAZÔNIA
}

\author{
SYSTEMATIZATION OF NURSING CARE FOR PEDIATRIC ONCOLOGICAL \\ PATIENTS IN A PUBLIC HOSPITAL IN THE INTERIOR OF THE AMAZON
}

\author{
Márcia Daiane Sousa Ribeiro ${ }^{1}$ \\ Raclice Silva da Cruz ${ }^{2}$ \\ Thaianna Cristina Oliveira Imbiriba ${ }^{3}$
}

RESUMO: INTRODUÇÃO: O câncer infantil é definido como um conjunto de grupos de patologias distintas com diferentes causas, manifestações, tratamentos e prognósticos. O exercício da enfermagem oncológica, dentro de uma proposta estabelecida pela equipe multiprofissional tornou-se mais abrangente e complexa, uma vez que a assistência promove cuidados a saúde de forma fundamentada e organizada. O enfermeiro tem de estabelecer uma relação com paciente e família, por meio da comunicação efetiva, humanizando a assistência, garantindo o controle dos sintomas, medidas para alívio do sofrimento e apoio aos familiares no processo do tratamento. OBJETIVO: O presente estudo objetivou descrever a sistematização da assistência de enfermagem ao paciente pediátrico oncológico em um hospital regional no interior da Amazônia, na cidade de Santarém-Pará. MATERIAIS E MÉTODOS: Caracteriza-se uma pesquisa de campo, com abordagem quantitativa que busca investigar e descrever a sistematização da assistência de enfermagem ao paciente pediátrico oncológico. RESULTADOS E DISCUSSÕES: Os resultados mostraram, maior índice dentre crianças com idade entre o8 aio anos e maior índice para o sexo masculino de pacientes diagnosticado com câncer. Evidenciou-se também que com relação a análise dos diagnósticos de enfermagem-DE mais frequentes, obteve-se uma frequência total de 7r ocorrência de registros de DE, sinalizando mais de um Diagnóstico de Enfermagem por paciente. E com relação às prescrições de enfermagem mais frequentes, a pesquisa mostrou que as intervenções de enfermagem propostas para os diagnósticos identificados, foram elaboradoras ou estão de acordo com o que é proposto a partir de cada necessidades básicas, proposta pelo NIC. CONCLUSÃO: Portanto, o uso da Sistematização de Assistência de Enfermagem-SAE, são fatores que contribuem para o desenvolvimento de uma sistematização adequada e com qualidade, relatando a importância do enfermeiro sobre tais conhecimentos e habilidades, pois a utilização da SAE garante contribuir e

\footnotetext{
I Acadêmica do curso de bacharelado em Enfermagem pelo Centro Universidade da AmazôniaUNAMA. E-mail: marciadaiane2ro8@gmail.com.

${ }^{2}$ Acadêmica do curso de bacharelado em Enfermagem pelo Centro Universidade da AmazôniaUNAMA. E-mail: raclicesilva@gmail.com.

${ }^{3}$ Orientadora Enfermeira Especialista em Saúde Coletiva do Centro Universitário da AmazôniaUNAMA.e-mail: thaianna.imbiriba@hotmail.com.
} 
nortear os cuidados e as intervenções de enfermagem, de acordo com as necessidades do paciente, com foco na humanização da assistência, proporcionando melhor qualidade no cuidado.

Palavras-chaves: Processo de enfermagem. Cuidados de Enfermagem. Oncologia.

ABSTRACT: INTRODUCTION: Childhood cancer is defined as a set of distinct pathologies groups with different causes, manifestations, treatments and prognoses. The exercise of oncologicaldisease, withina proposal established by the multidisciplinary team has become more comprehensiveand complex, since care promotes health care in a reasoned and organized way. Nurses must establish a relationship with patients and families through effective communication, humanizing care, ensuring the control of symptoms, measures to relieve suffering and support family members in the treatment process. OBJECTIVE: This study aimedtodescribe the systematization of nursing care to pediatric cancer patients in a regional hospital in the interior of the Amazon, inthe city ofSantarém-Pará. MATERIALS AND METHODS: A field research is characterized, with a quantitative approach that seeks to investigate and describe the systematization of nursing care to pediatric cancer patients. RESULTS AND DISCUSSIONS: The results showed a higher rate among children aged between o8 and ro years and higher rate for males of patients diagnosed with cancer. It was also evidenced that in relation to the analysis of the most frequent nursing-ED diagnoses, a total frequency of 71 occurrence of ED records was obtained, signaling more than one Nursing Diagnosis per patient. And regarding the most frequent nursing prescriptions, the research showed that the nursing interventions proposed for the identified diagnoses were elaborate or are in accordance with what is proposed from each basic needs, proposed by the NIC. CONCLUSION: Therefore, the use of the Systematization of Nursing Care-SAE, are factors that contribute to the development of an adequate and quality systematization, reporting the importance of nurses on such knowledge and skills, because the use of THE ensures to contribute andguide nursing care and interventions, according to the needs of the patient, focusing on humanization ofcare, providing better quality in care.

Keyword: Nursing Process. Nursing Care. Medical Oncology.

\section{INTRODUÇÃO}

De acordo com o Instituto Nacional do Câncer-INCA (2021), o câncer infantojuvenil se refere a um grupo de várias doenças que compartilham de uma característica em comum, que é uma proliferação descontrolada de células anormais que podem ocorrer em qualquer parte do corpo. As neoplasias infantis mais comumente na infância e adolescência são as leucemias, que atingem as células de 
defesa do corpo (glóbulos brancos), tumores do sistema nervoso central e linfomas que se desenvolvem no sistema linfático.

Para Vieira; Castro; Coutinho (2016), afirmam que com o diagnóstico de câncer, o cotidiano das crianças e de suas famílias passa por enormes mudanças. Inicialmente, a internação é necessária para definir e iniciar o tratamento, o que pode levar muito tempo. Com isso, há um afastamento do convívio social, escolar, amigos e familiares, uma vez que, a criança entra em um ambiente até então desconhecido, repleto de procedimentos invasivos, acompanhados de reações indesejadas.

A equipe de enfermagem é uma das categorias profissionais em que a criança com câncer e seus pais têm maior contato durante o acompanhamento oncológico. Para trabalhar na oncologia pediátrica, os profissionais de enfermagem, necessitam de uma ampla capacitação de conhecimento e compreensão dos aspectos, tanto biológicos, físicos, sociais, quanto emocionais da criança. Além disso, o acolhimento, o vínculo, a escuta, a paciência e o respeito à criança e sua família são essenciais para prestar uma ajuda humanizada, afim de tomar o tratamento oncológico menos doloroso e traumático. (SILVA et al. 2018).

Diante isso, Massaroli et al. (2015) destacam que a Sistematização da Assistência de Enfermagem-SAE é um instrumento que promove a prática do profissional enfermeiro. A sua implementação no cotidiano de trabalho auxilia a compreender a importância das ações organizacionais, utilizando meios adequados à sua realidade e ajuda na tomada de decisões.

Em consonância com a Resolução COFEN 358/2009, sua implantação é uma exigência para os estabelecimentos de saúde, públicos e privados, em todo o Brasil, sendo que a SAE é exclusiva da equipe de enfermagem (COFEN, 2009).

Com tudo, o interesse pela realização desse estudo deve-se ao fato de que para que os esforços da enfermagem oncológica sejam abrangentes e resolutivos, os profissionais de enfermagem devem possuir conhecimentos técnico-científicos e habilidades interpessoais, favorecendo as práticas na detecção e contribuição para a tratamento do câncer, com enfoque na participação da enfermagem em prestar uma assistência de qualidade e sistematizada ao paciente oncológico pediátrico. 
Assim, espera-se contribuir para a maior compreensão dos cuidados de enfermagem que são prestados às crianças hospitalizadas para tratamento oncológico, com o objetivo de estimular a reflexão e o desenvolvimento de novas práticas que ofereçam uma assistência de enfermagem mais humanizada e de qualidade à criança em tratamento oncológico.

\section{OBJETIVO GERAL}

Analisar a eficácia da aplicabilidade prática da Sistematização da Assistência de Enfermagem-SAE nos cuidados prestados aos pacientes pediátricos oncológicos.

\section{I Objetivos específicos}

$\checkmark$ Identificar o nível de conhecimento e habilidades da equipe de enfermagem quanto a utilização da SAE no cuidado aos pacientes pediátricos oncológicos;

$\checkmark$ Analisar os principais diagnósticos e intervenções de enfermagem aplicados no cuidado diário dos pacientes pediátricos oncológicos com a finalidade de garantir uma assistência de qualidade e humanizada.

\section{METODOLOGIA}

O presente estudo caracteriza-se como uma pesquisa descritiva, de campo, bibliográfico com abordagem do tipo quantitativa que busca investigar e analisar a eficácia da aplicação na prática da Sistematização da Assistência de EnfermagemSAE nos cuidados prestados aos pacientes pediátricos oncológicos na Clínica Oncológica Pediátrica.

De acordo com Corrêa (2018), a pesquisa descritiva tem o objetivo de observar, registrar e analisar com detalhes um objetivo de estudo, ou seja, o pesquisador apenas irá descobrir a frequência com que o fenômeno acontece sem adultera-las.

Para Silva (2014) a pesquisa quantitativa tem seu foco voltado na objetividade, o método caracteriza-se pelo emprego da quantificação, tanto nas modalidades de coleta de informações, quanto no tratamento delas por meio de técnicas estatísticas. 
A pesquisa foi realizada no mês de setembro de 2021 na Pediatria do Hospital Regional do Baixo Amazonas-HRBA, localizado no município de Santarém-PÁ, é uma unidade de saúde pública de referência do Oeste do Pará pertencente ao Governo do Pará e administrado pela Organização Social Pró-Saúde Associação Beneficente de Assistência Social e Hospitalar. Está localizado na Avenida Sérgio Henn, no ııo, Bairro - Diamantino - Santarém-Pará.

A população desta pesquisa foi composta por 25 prontuários de pacientes com diagnóstico de câncer na idade de 5 a 12 anos que receberam tratamento no período de 2018 a 2020. Neste estudo, foi utilizado os seguintes critérios de inclusão: prontuários de pacientes que foram admitidos com diagnóstico de câncer que estejam na faixa etária entre 5 e 12 anos e que receberam tratamento nos anos de 2018 a 2020 na pediatria do Hospital Regional do Baixo Amazonas - HRBA no município de Santarém.

Foram excluídos da pesquisa: prontuários de pacientes que foram admitidos com Diagnóstico de Câncer menores de 5 anos e maiores de 12 anos, e com informações incompletas para compor a pesquisa, bem como, aqueles que estavam na clínica para tratamento para outras patologias afins.

Os dados utilizados na pesquisa foram obtidos por meio da análise dos prontuários de pacientes com diagnóstico de câncer na faixa etária entre 5 a 12 anos, que receberam tratamento no período de 2018 a 2020, contendo as práticas realizadas pela equipe de enfermagem. Para realizar a coleta de dados foi utilizado um questionário fechado onde as perguntas foram pautadas na avaliação da Sistematização de Assistência de Enfermagem - SAE ao paciente pediátrico oncológico, totalizando II questões evidenciando a problemática em questão.

Os questionários impressos foram aplicados, os quais foram identificados através de códigos alfanuméricos para identificação do participante, preservando com isso a confidencialidade das informações prestadas.

\section{RESULTADOS E DISCUSSÃO}

Foram analisados 25 prontuários dos pacientes pediátricos oncológicos que receberam tratamento na Clínica Pediátrica no período de 2018 a 2020 do Hospital 
Regional do Baixo Amazonas situado no município de Santarém - Pará no mês de setembro de 2020. Os dados utilizados na pesquisa foram oriundos de prontuários de pacientes da Pediatria, mediante a aceitação do termo de compromisso para a utilização de dados (TCUD) para participar do estudo.

No gráfico I demonstra a distribuição da idade dos pacientes de acordo com o questionário. Observou-se que as crianças na faixa etária de o8 a ro anos obtiveram o maior índice dentre as faixas etárias de idade de pacientes que foram internados na Pediatria com diagnóstico de câncer.

Os resultados mostraram que de 25 dos prontuários de pacientes diagnosticados com câncer na Pediatria, aqueles com idade entre o8 a io anos correspondem a 48,0\% ( 12 prontuários) do total de prontuários analisados, e 44,0\% (II prontuários) do total de prontuários analisados estão os pacientes com idade entre o5 a 07 anos, e na idade entre II a 12 anos correspondem a 8,0\% (2 prontuários) do total de prontuários analisados.

No que se refere a faixa etária, os resultados do estudo estão em discordância aos encontrados por Diniz et al. (2005), realizado na Unidade de Oncologia Pediátrica em Salvador - BA, afim de descrever os aspectos epidemiológicos, demográficos e possíveis fatores de risco, genéticos e possíveis fatores de risco, genéticos e ambientais do câncer infantil, no qual identificou que o câncer foi mais frequente em crianças na idade entre um a quatro anos $(32,5 \%)$.

Do mesmo modo, um estudo realizado por Nascimento et al. (2020) sobre Perfil de pacientes Atendidos na Unidade de Alta Complexidade em Oncologia (UNACON) em Rio Branco - Acre, Brasil, no ano de 2017, identificaram que a maior patê dos pacientes tinha de um a três anos $(45,0 \%)$.

\section{Gráfico I:}

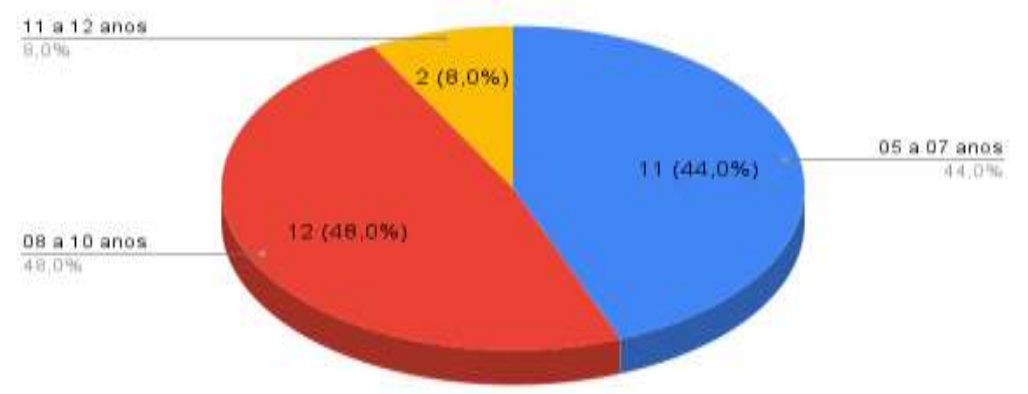


Distribuição da idade dos pacientes internado na Pediatria.

Fonte: Dados da pesquisa no Hospital regional do Baixo Amazonas - HRBA, 202I.

Em relação ao sexo (gráfico 2), a pesquisa mostrou que os dos pacientes internados na Pediatria em tratamento para câncer, 60,0\% (I5 prontuários) do total de prontuários analisados eram do sexo masculino, quanto 40,0\% (Io prontuários) correspondem ao sexo feminino.

Gráfico 2: Distribuição do sexo dos pacientes internados na Pediatria.

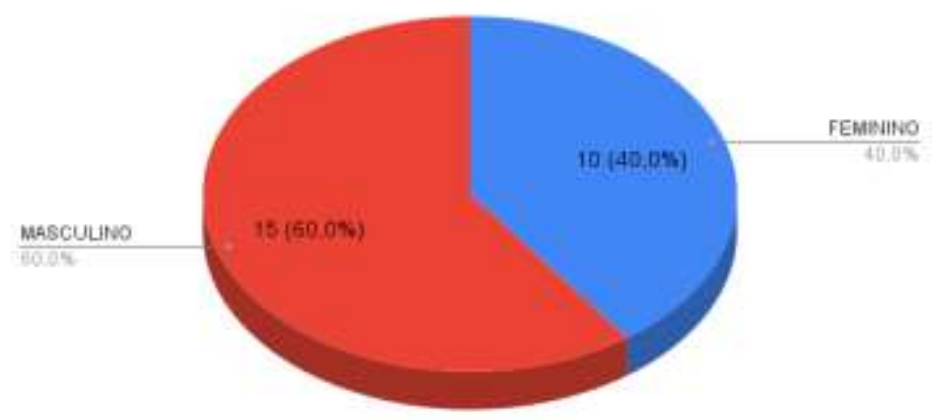

Fonte: Dados da pesquisa no Hospital regional do Baixo Amazonas - HRBA, 2021.

De acordo com Pan et al. (2011), na maioria das populações dos países em desenvolvimento, a ocorrência e mortalidade dos canceres infantis são evidenciados em sua maioria para o sexo masculino, desse modo, considera-se que há uma sustentabilidade maior do desenvolvimento das neoplasias pediátricas no sexo 
masculino, também, sendo identificado que os pacientes com maior tempo de hospitalização são aqueles do sexo masculino, quando comprado ao sexo feminino.

Corroborando com isso, Diniz et al. (2005), relata que o câncer infantil tem maior ocorrência no sexo masculino, portanto, apresenta maior potencial de debilidade ou uma associação a fatores genéticos de predisposição ligados ao cromossomo Y.

Quanto a análise dos diagnósticos na admissão dos pacientes na Clínica Pediátrica (tabela I), evidenciou-se que $9(37,5 \%)$ do total de prontuários analisados equivalem ao Diagnóstico de Leucemia Linfoblástica Aguda, 2(8,33\%) do total de prontuários analisados ambos correspondem aos Diagnósticos de Leucemia Mieloide Aguda e Neoplasias do Sistema Nervoso Central, Doença de Hodgkin, Neoplasias do Pulmão e Neoplasias dos Tecidos Moles da Cabeça. Já I (4,16\%) do total de prontuários analisados ambos correspondem aos diagnósticos: Neoplasia do ovário, Histiocitose maligna, Neoplasia do mediastino, fistula do intestino, Neoplasia da conjuntiva e Neoplasia do retroperitônio.

Gráfico 2: Distribuição do sexo dos pacientes internados na Pediatria.

\begin{tabular}{lcc}
\hline DIAGNÓSTICOS NA ADMISSÃO & N & $\%$ \\
\hline Leucemia Linfoblástica aguda & 9 & $37,5 \%$ \\
\hline Leucemia mieloide aguda & 2 & $8,33 \%$ \\
\hline Neoplasia do sistema nervoso central & 2 & $8,33 \%$ \\
\hline Linfoma de Hodgkin & 2 & $8,33 \%$ \\
\hline Neoplasia dos tecidos moles da cabeça & 2 & $8,33 \%$ \\
\hline Neoplasia do Pulmão & 2 & $8,33 \%$ \\
\hline Neoplasia do ovário & I & $4, \mathrm{I} 6 \%$ \\
\hline Histiocitose maligna & I & $4,16 \%$ \\
\hline Neoplasia do mediastino & I & $4,16 \%$ \\
\hline Fistula do intestino & I & $4,16 \%$ \\
\hline Neoplasia da conjuntiva & I & $4,16 \%$ \\
\hline Neoplasia do retroperitônio & \multicolumn{1}{c}{ I } & $4,16 \%$ \\
\hline & 25 & I00\% \\
\hline
\end{tabular}

Fonte: Dados da pesquisa no Hospital regional do Baixo Amazonas - HRBA, 2021.

Os cânceres em crianças e adolescentes têm características que os distinguem dos cânceres adultos. Originam-se principalmente de células embrionárias, tem um 
curto período de latência e, geralmente, cresce rapidamente. Em adultos, o principal fator de risco para o câncer são os fatores ambientais, que estão relacionados à exposição a agentes cancerígenos e hábitos de vida ruins, porém, nas crianças, já nas primeiras duas décadas de vida, o desenvolvimento do câncer está intimamente relacionado a fatores genéticos ou mutações adquiridas de causas incertas (BRASIL, 2017).

Segundo o INCA (2019), a Leucemia Linfoblástica Aguda - LLA é o câncer mais comum em crianças e adolescentes. Está relacionada a uma variedade de fatores genéticos, ambientais e maternos que aumentam o risco da doença. A maior incidência desse câncer se dá em crianças na idade entre dois e cinco anos, indicando um predomínio ligeiramente maior em indivíduos do sexo masculino.

De acordo com Negreiros et al. (2015) os tumores do sistema nervoso central (SNC) constituem o segundo grupo mais comum de neoplasias na infância, também, são os tumores mais frequentes e a principal causa de morte por câncer em jovens. Seguindo a mesma linha de pensamento, Oliveira; Campos (2015) relatam que os linfomas que são classificados como linfoma de Hodgkin (LH) e linfoma não Hodgkin (LNH), representam o terceiro tipo de câncer mais incidente na criança, sendo que o risco de desenvolvimento é maior com o aumento da idade.

Tabela 2: Distribuição dos diagnósticos de enfermagem mais freqüentes dos pacientes internados na Pediatria.

\begin{tabular}{|c|c|c|c|}
\hline $\begin{array}{l}\text { DOMÍNIO } \\
\text { /CLASSE }\end{array}$ & $\begin{array}{l}\text { DIAGNÓSTICOS } \\
\text { ENFERMAGEM }\end{array}$ & $\mathbf{N}$ & $\%$ \\
\hline D: II, C:I & Risco de infecção & 24 & $33,80 \%$ \\
\hline D:II, C:2 & Risco de criança cair & 19 & $26,76 \%$ \\
\hline D: II, C:6 & Risco de termorregulação ineficaz & 19 & $26,76 \%$ \\
\hline $\mathrm{D}: \mathrm{II}, \mathrm{C}: 2$ & Risco de integridade da pele prejudicada & 3 & $4,22 \%$ \\
\hline D:II, C:2 & Risco de lesão por pressão infantil & 2 & $2,8 \mathrm{I} \%$ \\
\hline $\mathrm{D}: 12, \mathrm{C}: \mathrm{I}$ & Dor aguda & 2 & $2,8 \mathrm{I} \%$ \\
\hline $\mathrm{D}: 4, \mathrm{C}: 5$ & Déficit no auto cuidado & 2 & $2,8 \mathrm{I} \%$ \\
\hline & $\Sigma$ & $7 \mathrm{I}$ & IOO\% \\
\hline
\end{tabular}

Fonte: Dados da pesquisa no Hospital regional do Baixo Amazonas - HRBA, 202I. 
Com relação a análise dos Diagnósticos de Enfermagem - DE mais frequentes (tabela 2), foram obtidas as seguintes informações: $24(33,80 \%)$ do total de prontuários analisados possuem o DE Risco de Infecção, I9 (26,76\%) possuem o DE Risco de Criança Cair e Risco de Termorregulação Ineficaz. O DE Risco de Integridade da Pele Prejudicada equivale a $3(4,22 \%)$ do total de prontuário. Os DE Risco de Lesão por Pressão Infantil, Dor aguda e Déficit no Autocuidado corresponde a $2(2,81 \%)$ do total de prontuários analisados. Dos 25 pacientes, obteve se uma frequência total de 7r ocorrência de registros de diagnósticos de enfermagem, sinalizando mais de um diagnóstico de enfermagem por paciente.

Para elencar os títulos diagnósticos do estudo, foi utilizado a Taxonomia II da Associação Norte Americana de Diagnósticos de Enfermagem - NANDA, que caracteriza - se por um sistema de classificação que organiza os DE com a finalidade de desenvolver uma terminologia padronizada de enfermagem para promover a melhora do cuidado prestado. A Taxonomia II da NANDA 202I - 2023 estrutura-se em 03 níveis: domínios, classes e diagnósticos de enfermagem. Sendo composta por 13 domínios, 47 classes e 267 diagnósticos de enfermagem (NANDA, 2021).

O domínio defini - se como sendo uma esfera de conhecimentos, influências e questionamentos, já a classe é um grupo ou conjunto que partilham de atributos comuns e o DE é um julgamento clinico sobre uma resposta humana a condições de adoecimento ou de vulnerabilidade individual, familiar ou da comunidade (NANDA, 2015).

$\mathrm{Na}$ década de 1970, Wanda de Aguiar Horta introduziu o Processo de Enfermagem - PE no Brasil, que trouxe como referência a Teoria das Necessidades Humanas Básicas - NHB de Maslow. Sendo assim, o PE é a força motriz de ações sistemáticas e interligadas, com a finalidade de prestar assistência ao ser humano, sendo estas exercidas pelo profissional de enfermagem (HORTA, 20II).

Desse modo, Souza et al. (2017) afirmam que o cuidar em enfermagem tem suas raízes sustentadas na teoria criada por Wanda de Aguiar Horta, que propôs uma metodologia de assistência baseada na Teoria das Necessidades Humanas Básicas NHB, que abrande a avaliação do indivíduo nos aspectos psicobiológicos, psicossociais e psicoespirituais. 
De acordo com a Resolução COFEN n 358/2009, que dispõem sobre a Sistematização da Assistência de Enfermagem - SAE e a implementação do Processo de Enfermagem - PE, em ambientes públicos e privados onde ocorrem o cuidado do profissional de enfermagem. Deve ser realizado em cinco etapas simultâneas e interrelacionadas, a saber: coleta de dados, diagnósticos de enfermagem, planejamento, implementação da assistência e avaliação, sendo exercido de acordo com as necessidades do indivíduo, assim, possibilitando a abordagem holística do paciente e o aperfeiçoamento continuo da assistência de enfermagem.

Para Silva (2017) a Sistematização da Assistência de Enfermagem (SAE) se define como sendo tudo aquilo que estrutura e organiza a atividade laboral do profissional enfermeiro, fundamentado em base teórico - filosófico que oportunize a implementação do Processo de Enfermagem - PE, sendo este, também, fundamentado em base teórico - filosófico.

Do mesmo modo, Gutierres et al. (2018) e Pereira et al. (2017) afirmam que a utilização da SAE é imprescindível para que o enfermeiro preste seus cuidados de forma segura, uma vez que, uma assistência sistematizada prove recursos tecnológicos e humanos ao enfermeiro, dessa maneira, melhora a qualidade dos cuidados prestados e propicia que o trabalho do profissional de enfermagem seja apreciado pela sociedade.

Porém, algumas dificuldades são encontradas em torno da SAE, Oliveira et al. (2019) afirmam que, embora ela seja um instrumento de assistência regulamentado e a existência de publicações cientificas sustentam sua eficácia, ainda é corriqueiro o relato de enfermeiros sobre as adversidades na execução da SAE na rotina diária do cuidar, tal como de compreende - lá, por muitos, como uma simples ferramenta de coleta de dados, não percebendo que a SAE vai além de uma mera atividade burocrática.

De acordo com Peterson e Carvalho (2011), descrevem que os cuidados de enfermagem para os pacientes com câncer, devem ser individualizados, tendo em vista que o paciente está fragilizado, diante do diagnóstico de uma doença neoplásica e com uma expectativa de vida reduzida. Deste modo, o enfermeiro encontra-se presente nas transformações fisiológicas e psíquicas, proporcionando uma maior 
aproximação com o paciente por intermeio da comunicação, além de identificar as necessidades e proporcionar uma melhor assistência e qualidade de vida para o paciente.

Corroborando com esse pensamento Castro et a. (2017) relata que o diagnóstico de câncer representa um alto grau de complexidade do cuidado, em linhas gerais, é de responsabilidade da enfermagem oncológica garantir um cuidado humanizado e especializado, uma vez que, o paciente está diante de uma possível cura ou condições incuráveis. Sendo assim, a utilização da SAE potencializa a compreensão das necessidades dos indivíduos com neoplasias malignas e pode se tornar uma ferramenta para maximizar o atendimento aos pacientes com especificidades.

Com relação as prescrições de enfermagem mais frequentes (Tabela 3), a pesquisa mostrou que as intervenções de enfermagem propostas para os diagnósticos identificados visaram: proteção contra infecções, prevenção contra quedas, precauções contra aspiração, precauções com a integridade da pele, prevenção de úlceras por pressão, prevenção de lesões na pele, prevenções a reações alérgicas, promoção do equilíbrio da temperatura corporal, e prevenção de complicações.

Ao que se refere as Prescrições de Enfermagem - PE mais frequentes (Tabela 3), a partir da análise dos prontuários, identificou - se que as intervenções de enfermagem foram em sua maioria para a prevenção de infecções, sendo elas a higienização das mãos $(\mathrm{N}=24)$, avaliação de sinais de infecção no sítio de inserção $(\mathrm{N}=24)$, troca de curativo e lavagem com SF $0,9 \%(\mathrm{~N}=24)$ correspondendo a $10,08 \%$ (24 prontuários), devido à grande labilidade de risco de infecção. Também, foi possível visualizar a prevenção de risco de queda, a partir do controle da elevação de grades e rodas travadas $(\mathrm{N}=19)$, orientar chamada da enfermagem para auxiliar deambulação $(\mathrm{N}=19)$ e ronda no leito $(\mathrm{N}=19)$, uma vez que, a criança está em constante risco de cair devido a idade precoce, correspondendo a 7,98\% identificados em 19 do total de prontuários analisados.

Tabela 3: Principais intervenções de enfermagem implementadas aos pacientes internados na Pediatria.

\begin{tabular}{lcc}
\hline PRESCRIÇÕES DE ENFERMAGEM & N & $\%$ \\
\hline Higienização das mãos & 24 & $10,08 \%$ \\
\hline Avaliar sinais de infeç̧ão no sitio de inserção & 24 & $10,08 \%$ \\
\hline
\end{tabular}




\begin{tabular}{|c|c|c|}
\hline Troca de curativo & 24 & 10,08\% \\
\hline Lavagem com SF o,9\% & 24 & $10,08 \%$ \\
\hline Manter grades elevadas e rodas travadas & 19 & $7,98 \%$ \\
\hline Orientar chamada da enfermagem para auxiliar deambulação & 19 & $7,98 \%$ \\
\hline Ronda no leito & 19 & $7,98 \%$ \\
\hline $\begin{array}{l}\text { Realizar compressas em região frontal e cervical de temperatura maior que } \\
39^{\circ} \mathrm{C}\end{array}$ & 19 & $7,98 \%$ \\
\hline Verificar sinais vitais & 19 & $7,98 \%$ \\
\hline Administrar antitérmico prescrito se temperatura maior que $37,8^{\circ} \mathrm{C}$ & 19 & $7,98 \%$ \\
\hline Monitorar pele e as mucosas quanto aos sinais de infecção & 3 & $1,26 \%$ \\
\hline Checar permeabilidade do acesso para administração do quimioterápico & 3 & $\mathrm{I}, 26 \%$ \\
\hline Identificar sinais de extravasamento do AVP & 3 & $\mathrm{I}, 26 \%$ \\
\hline Hidratação da pele & 2 & $0,84 \%$ \\
\hline Manter lençóis sempre alinhados & 2 & $0,84 \%$ \\
\hline Manter placa de hidrocoloide ou coxins nas proeminências ósseas & 2 & $0,84 \%$ \\
\hline Realizar mudança de decúbito de $2 / 2 \mathrm{~h}$ & 2 & $0,84 \%$ \\
\hline Realizar higiene oral $3 \mathrm{x}$ ao dia & 2 & $0,84 \%$ \\
\hline Realizar banho no leito $2 \mathrm{x}$ ao dia & 2 & $0,84 \%$ \\
\hline Auxiliar na deambulação & 2 & $0,84 \%$ \\
\hline Avaliar dor e intensidade de $2 / 2 \mathrm{~h}$ e comunicar ao enfermeiro & 2 & $0,84 \%$ \\
\hline Avaliar nível de consciência e respiração após medicação & 2 & $0,84 \%$ \\
\hline$\Sigma$ & 237 & $100 \%$ \\
\hline
\end{tabular}

Fonte: Dados da pesquisa no Hospital regional do Baixo Amazonas - HRBA, 2021.

As prescrições de enfermagem para o controle de desequilíbrios na temperatura corporal, estão a realização de compressas em região frontal e cervical $(\mathrm{N}=19)$ e verificação de sinais vitais $(\mathrm{N}=19)$ que correspondem a $7,98 \%$ （9 prontuários). Já no que tange aos cuidados voltados para a prevenção de lesões na pele, estão as prescrições: monitorar pele e mucosas quanto aos sinais de infecção $(\mathrm{N}=3)$, checar permeabilidade do acesso para administração do quimioterápico $(\mathrm{N}=3)$ e identificar sinais de extravasamento do acesso venoso periférico - AVP $(\mathrm{N}=3)$ que corresponderam a I, $26 \%$ ( 3 prontuários), o que se justifica pelo alto risco de lesões na pele devido ao tratamento quimioterápico.

Já no que se refere a prevenção do desenvolvimento de lesões por pressão, as PE identificadas no estudo foram a hidratação da pele $(\mathrm{N}=2)$, manter lençóis sempre alinhados $(\mathrm{N}=2)$, manter placa de hidrocoloide nas proeminências ósseas $(\mathrm{N}=2)$ e realizar mudança de decúbito de $2 / 2 \mathrm{~h}(\mathrm{~N}=2)$, uma vez, a criança hospitalizada está em risco de desenvolver lesões por pressão devido ao longo tempo restrito ao leito, que equivaleram a $0,84 \%$ sendo identificadas em 3 do total de prontuários analisados. 
Ademais, no que se refere aos cuidados de enfermagem a criança oncológica com dor, as principais prescrições de enfermagem identificadas foram a avaliar o nível de consciência e respiração após medicação $(\mathrm{N}=2)$ e avaliar dor e intensidade de 2/2h $(\mathrm{N}=2)$, com $0,84 \%$, no qual foram mencionados em dois do total de prontuários analisados. Desse modo, percebe - se que a dor é um dos principais sinais em que o enfermeiro deve voltar sua atenção e cuidados, afim de garantir a qualidade da assistência.

Ainda, para aquelas crianças que apresentaram déficits no auto cuidado, a dificuldade ou a impossibilidade de realizar atividades de vida diária simples, foi identificado as prescrições de enfermagem no que diz respeito ao auxilio à criança em realizar higiene oral $3 \mathrm{x}$ ao dia $(\mathrm{N}=2)$, realizar banho no leito $2 \mathrm{x}$ ao dia $(\mathrm{N}=2)$ e auxiliar na deambulação $(\mathrm{N}=2)$ que corresponderam a $0,84 \%$, sendo observados em dois do total de prontuários analisados.

Foi utilizado a Classificação das Intervenções de Enfermagem - NIC (2010), para descrever as prescrições de enfermagem identificadas no estudo, que é um método que classifica as intervenções de enfermagem, independentes ou colaborativas, de cuidados direto ou indireto. Uma vez que a intervenção de enfermagem é determinada como um meio de tratamento, realizado pelo enfermeiro e fundamentado no julgamento e no conhecimento clínico para aperfeiçoar os resultados do paciente.

Corroborando com isso, Bulechek (20II), afirma que o NIC, trata do cuidado direto ou indireto, com foco no indivíduo, família e comunidade, é o arranjo das atividades de cuidado, de acordo com o seu grupo ou acervo, levando em consideração a relação e decisão da intervenção grupal designada. A escolha das intervenções requer que os enfermeiros tomem decisões diretas para pacientes específicos.

Segundo Santos et al. (2016) a criança hospitalizada passa por situações estressantes e traumatizantes, uma vez que, está em um ambiente farto de restrições e rotinas, em que a criança fica exposta procedimentos que geram medo ansiedade e dor. Por essa razão, a criança sofre um afastamento de seu meio social, costumes, atividades e hábitos de rotina. Ao se falar de tratamento oncológico, essa repercussão 
pode ser ainda mais grave, dado que, é necessário que a criança permaneça internada por um longo período de tempo.

Ainda nessa linha de pensamento, Silva et al. (2019), relata que o tratamento do câncer infantil, devido ao longo período de tempo de internação, ocasiona traumas à criança, seus familiares e aos profissionais de saúde. Apesar da utilização de todos os recursos técnicos curativos, em todos os casos possíveis de cura ou não, a criança certamente sofrerá dores psicológicas, sociais, espirituais e físicas durante o tratamento.

Corroborando com isso, Silva et al. (2014), afirma que a assistência de enfermagem em oncologia pediátrica vai além da assistência técnica. A especificidade de cada família deve ser levada em consideração, levando em conta a multidimensionalidade dessa criança e de seus responsáveis. O diálogo se torna uma ferramenta necessária para a eficácia do cuidado seguro às crianças, de forma a reduzir os riscos à sua saúde.

Desse modo, para Silva et al. (2018) o cuidado humanizado é uma parte importante do cuidado da criança em tratamento contra o câncer. Sendo assim, a equipe de enfermagem deve se empenhar em oferecer atendimento de qualidade e eficácia ao paciente e seus familiares, tornando o tratamento menos traumático e mais agradável.

Em virtude disso, a implementação da Sistematização da Assistência de Enfermagem - SAE se faz necessária para alcançar e garantir padronização dos cuidados e serviços de enfermagem ao paciente pediátrico oncológico. No entanto, são necessárias habilidades assistenciais e gerenciais, uma vez que esta metodologia representa uma revolução na forma de prestar os serviços de saúde, favorecendo a reorganização de recursos físicos, humanos e administrativos, além de ser uma metodologia que direciona às ações do enfermeiro (SANTANA, et al, 2013).

\section{CONSIDERAÇÕES FINAIS}

À medida em que aumenta a incidência do desenvolvimento do câncer infantil, torna-se necessário o aprimoramento da assistência, deste modo, a padronização da assistência auxilia no cuidado prestado à criança, logo, o profissional 
de enfermagem deve estar preparado, capacitado e possuir pensamento crítico para obter resultados satisfatórios na execução de suas atividades, garantindo o bem-estar do paciente pediátrico oncológico.

A realização do estudo evidenciou a importância da aplicação da Sistematização da Assistência de Enfermagem - SAE, de forma individualizada e humanizada a criança com câncer, resultando em um cuidado qualificado e contribuindo para a melhora continua da eficácia e segurança dos cuidados prestados pela equipe de enfermagem, bem como, documenta a prática profissional para que outros tenham acesso ao plano assistencial. Como também, fortalece a autonomia do enfermeiro na execução de suas ações e contribui para o enriquecimento da identidade profissional.

O fato de identificar a ocorrência de Diagnósticos de Enfermagem - DE tanto nos artigos obtidos nos bancos de dados online, bem como, nos dados coletados nos prontuários, sustentam a legitimidade da pesquisa, assegurando que os DE coletados na pesquisa também existem na literatura nacional sobre DE em pediatria.

Com base nos resultados deste estudo, esperamos que possamos contribuir para a qualidade e melhoria da assistência de enfermagem voltadas a criança com câncer e seus familiares, também, que venha servir de estímulo para novos estudos nesta área e, fornecer subsídios científicos para a implementação da Sistematização da Assistência de Enfermagem - SAE com qualidade e segurança ao paciente com câncer infantil, buscando não apenas a cura, mas também a garantia da qualidade de vida e o melhor prognóstico do paciente pediátrico oncológico.

\section{REFERÊNCIAS BIBLIOGRÁFICAS}

BRASIL. Ministério da Saúde. Secretaria de Atenção à Saúde. Departamento de Atenção Especializada e Temática. Protocolo de diagnóstico precoce para oncologia pediátrica [recurso eletrônico] / Ministério da Saúde, Secretaria de Atenção à Saúde, Departamento de Atenção Especializada e Temáticas - Brasília: Ministério da Saúde, 2017. Disponível em: https://bvsms.saude.gov.br/bvs/publicacoes/protocolo_diagnostico_precoce_cancer_ pediatrico.pdf, aceso em: 20/10/2021.

BULECHEK MG, Butcher KH, Dochterman MJ. Classificação das Intervenções de Enfermagem (NIC). sa ed. Mosby/Elsevier; 2011. 
Conselho Federal de Enfermagem (COFEN). Resolução COFEN no 358/2009. Dispõe sobre a Sistematização da Assistência de Enfermagem e a implementação do Processo de Enfermagem em ambientes, públicos ou privados, em que ocorre o cuidado profissional de Enfermagem, e dá outras providências. Brasília: COFEN; 2009. Disponível em: http://www.cofen.gov.br/resoluo-cofen-3582009_4384.html , acesso em: 26/10/2021.

CORREA, Luiz Nilton. Metodologia Científica: Para trabalhos acadêmicos e artigos científicos. Ir ed. Florianópolis/SC: Do Autor, 2017.

DINIZ, A. B. et al. Perfil epidemiológico do câncer infantil em população atendida por uma unidade de oncologia em Salvador-Bahia. R. Ci. med. biol, v. 4, n. 2, p. I31139, 2015 .

INCA. Câncer Infanto-juvenil. 2019. Disponível em: https://www.inca.gov.br/tipos-decancer/cancer-infantojuvenil , acesso em: 2I/o2/2021.

INCA. Câncer Infanto-juvenil. 2019. Disponível em: https://www.inca.gov.br/tipos-decancer/cancer-infantojuvenil , acesso em: 21/02/2021.

GUTIERRES LS, Santos JLG, Peiter CC, Menegon FHA, Sebold LF, Erdmann AL. Good practices for patient safety in the operating room: nurses' recommendations. Rev Bras Enferm [Internet]. 2018;7I(Suppl 6):2775-82. [Thematic Issue: Good practices in the care process as the centrality of the Nursing]. Disponível em: doi: 10.1590/o034-7167-2018-0449, acesso em: 20/II/2021.

MASSAROLI R, et al. Trabalho de enfermagem em unidade de terapia intensiva e sua interface com a sistematização da assistência. Esc Anna Nery 19(2) Abr-Jun 2015. Disponível em: https://doi.org/I0.5935/1414-8145.20150033, acesso em: 26/10/2021.

NASCIMENTO, A. S. M.; NOBRE, I. C.; SOUZA LIMA, M. de F. de; ARRUDA, E. F. de; VOLPÁTI, N. V. Câncer infantojuninil: Perfil dos pacientes atendidos na unidade de alta complexidade em oncolologia (UNACON) em Rio Branco - Acre, Brasil, no ano de 2017. Arq. Cienc. Saúde UNIPAR, Umuarama, v. 24, n. I, p, 35-39, jan./abr. 2020. Disponível em: https://doi.org/10.25110/arqsaude.v24ir.2020.6898, acesso em: 26/10/2021.

Nanda International. Diagnósticos de Enfermagem da NANDA: definições e classificação 2021-2023. 12a edição. Herdman, T. Heather. Artmed; 2022. Disponível em: https://pt.scribd.com/document/531212468/NANDA-21Enfermagem-eDiagnosticos-202I-2023, acesso em: IO/II/202I.

Nanda International. Diagnósticos de Enfermagem da NANDA: definições e classificação 2018-2021. II ${ }^{\text {a }}$ edição. Porto Alegre: Artmed; 2018. Disponível em: http://www.podiatria.com.br/uploads/trabalho/I49.pdf, acesso em: Io/II/202I. 
Nanda International. Diagnósticos de Enfermagem da NANDA: definições e classificação 2015-2017. Tradução: Regina Machado Garcez. Io ${ }^{\mathbf{a}}$ ed. Porto Alegre: Artmed; 2015. Disponível em: https://www.biosanas.com.br/uploads/outros/artigos_cientificos/ı/df7Id2977c3c9e ab8c6c511a8692c2ad.pdf, acesso em: 02/II/2021.

NEGREIROS AV, et al. Aspectos Epidemiológicos dos Tumores do Sistema Nervoso Central Pediátricos em um Hospital de Referência de João Pessoa (PB) entre 2009 e 20II. Revista Acadêmica do Centro de Ciência Médicas da Universidade Federal da Paraíba, 2015;I(I): 51-59. Disponível em: https://www.semanticscholar.org/paper/Aspectos-Epidemiol\% $\mathrm{C}_{3} \% \mathrm{~B}_{3}$ gicosdosTumores-do-Sistema-em-

NegreirosMonteiro/bdf 4 cb392856de96afoe8r35eebe335e54895er7, acesso em: 26/10/2021.

NIC - Nusing Interventions Classification. Ed 5. Rio de Janeiro. 2010. Disponível em:https://www.biosanas.com.br/uploads/outros/artigos_cientificos/r4/oac40ssbe9 ao7e3df54c72e965Ic589e.pdf, acesso em: 02/ II/ 2021.

OLIVEIRA, André Luiz Gomes. DIAGNOSTICOS E INTERVENÇÕES DE ENFERMAGEM EM PEDIATRIA: MANUAL DE ORIENTAÇÃO. Universidade Federal Fluminense/UFF. Niterói, RJ. 2015. Disponível em: https://app.uff.br/riuff/handle/I/I72I, acesso em: 02/II/202I.

OLIVEIRA MCLA, CAMPOS MK. Linfomas em Pediatria. Pediatria Moderna, 2015; 5I(5): I73-180. Disponível em: https://pesquisa.bvsalud.org/portal/resource/pt/lil-754778, acesso em: 02/II/202I. PAN, R.; MARQUES, A. R.; JÚNIOR, M. L. C; NASCIMENTO, L. C. Caracterização das internações hospitalares de crianças e adolescentes com neoplasias. Revista Latino-Americana de Enfermagem, Ribeirão Preto, v. 19, n. 6, p. I413- I420, 2011. Disponível em: https://doi.org/I0.1590/Solo4-I169201100060oor9, acesso em: 2I/II/202I.

PAULA, D. P. S. et al. Câncer infantojuvenil do âmbito familiar: percepções e experiências frente ao diagnóstico. Revista Cuidarte, v. Io, n.I, p. I-I2, 2019.

PEREIRA GN, Abreu RNDC, Bonfim IM, Rodrigues AMU, Monteiro LB, Sobrinho JM. Relação entre sistematização da assistência de enfermagem e segurança do paciente. Enferm Foco. 2017; 8 (2): 21-5. Disponível em: doi: I0.21675/2357-707X.2017.v8. n2.985, acesso em: 20/II/2021.

PEROVANO, D, G. Manual de metodologia cientifica. Paraná: Editora Juruá, 2014. SANTANA et al. O CUIDAR EM ONCOLOGIA PEDIÁTRICA: UM ESTUDO BASEADO NO PROCESSO DE ENFERMAGEM. Revista Destaques Acadêmicos, Lajeado, v. 9, n. 3, 2017. Disponível em: http://dx.doi.org/I0.22410/issn.2176-3070.v9i3a2017.1511, acesso em: 26/10/2021. 
SANTANA, et al. Percepção dos enfermeiros acerca da Sistematização da Assistência de Enfermagem na atenção básica de Belo Horizonte. Revista. Enfermagem Revista. v. I6. n. I. jan./abr. 2013. Disponível em:< http://periodicos.pucminas.br/index.php/enfermagemrevista/article/view/12936>, acesso em: 20/10/2021.

SANTOS, P.M; SILVA, L.F; et al. Os cuidados de enfermagem na percepção de enfermagem da criança hospitalizada. Revista Brasileira de Enfermagem REBEN: São Paulo, v69, n4, p. 646-53, 2016. Disponível em: http://www.scielo.br/pdf/reben/v69n4/oo34-7167-reben-69-04-o646.pdf, acesso em: I8/10/2021.

SILVA, A.J.H. Metodologia da Pesquisa: Conceitos Gerais. UNICENTRO, PARANÁ. 2014. Acesso em: $\mathrm{I} 4$ de agosto de 2021. Disponível em: http://repositorio.unicentro.br:8o8o/jspui/bitstream/I23456789/84I/I/Metodologiada- pesquisa-cient $\% \mathrm{C}_{3} \% \mathrm{ADf}$ fica-conceitos-gerais.pdf.

SILVA, T., Leite, J., Santos, N., Silva, Í., Mendonça, A., Santos, M., \& Silva, L. Cuidados de Enfermagem à Criança com Câncer: Uma Revisão integrativa da Literatura. Revista de Enfermagem UFSM., Minas Gerais, v.3, n.I, p.68-78. Janeiro. 2013. Disponível em: https://doi.org/10.5902/217976926918 . Acesso em: 21/10/2021

SILVA; GARANHANI; GUARIENTE 2014 et al Sistematização da Assistência de Enfermagem: Aplicabilidade da Prática na Clínica Médica de um hospital do Interior. Artigo, Volume i8, número. João Pessoa, 2018. Disponível em: https://temasemsaude.com/wp-content/uploads/2018/o4/r8119.pdf Acesso em: I2/10/2021.

SILVA MCN. Sistematização da assistência de Enfermagem: desafio para a prática profissional. Enferm Foco. 2017;8(3). Disponível em: doi: 10.21675/2357707X.2017.v8.n3.1534, acesso em: 20/II/2021.

SILVA, Resende et al. Intervenções de enfermagem nos cuidados paliativos em Oncologia Pediátrica: revisão integrativa. Revista Brasileira de Enfermagem, v. 72, n. 2, 2019. Disponível em: https://www.scielo.br/pdf/reben/v72n2/pt_0034-7167-reben72-02-0531.pdf, acesso em: 24/10/2021.

SOUZA MAR, Wall ML, Chaves ACM, Lima DM, Santos BA. Poder vital e o legado de florence nightingale no processo saúde doença: revisão integrativa. Revista Pesqui Cuid é Fundam Online. 2017; 9(I):297.

STELIAROVA-FOUCHER Eva et al. Incidência internacional de câncer infantil, 200I-IO: um estudo de registro de base populacional. Vol. 4, junho de 2017. Disponível em: https://doi.org/10.1016/si470-2045(17)30186-9, acesso em: 26/10/2021. 
VIEIRA, A. P. M. S.; CASTRO, D. L.; COUTINHO, M. S. Assistência de enfermagem na oncologia pediátrica. Revista Eletrôn. Atualiza Saúde, Salvador, v. 3, n. 3, p. 67-75, jan./jun. 2016. 